\title{
Double Hopping: A new Approach for Dynamic Frequency Hopping in Cognitive Radio Networks
}

\author{
Daniel Willkomm, Mathias Bohge, Daniel Hollos, James Gross*, and Adam Wolisz \\ Telecommunication Networks Group, Technische Universität Berlin, Germany \\ Email: $\{$ willkomm|bohge|hollos|gross|wolisz $\} @$ tkn.tu-berlin.de
}

\begin{abstract}
One of the major challenges in designing cellular Cognitive Radio (CR) networks is the avoidance of Secondary User (SU) interference to so called Primary Users (PUs) operating in the licensed bands. Usually, SU operation has to be interrupted periodically in order to detect $P U$ activity and avoid the respective frequencies. Recently, Dynamic Frequency Hopping (DFH) mechanisms have been suggested to enable reliable $P U$ detection and continuous SU operation at the same time. Applying DFH in a multi-cell environment adds the challenge of mitigating Co-Channel Interference (CCI). In this paper, we introduce a new DFH approach for cellular CR networks to allow reliable PU detection and continuous SU operation while avoiding CCI: Double Hopping (DH). We present a distributed frequency assignment heuristic for DH and compare it to the optimal assignment. We show that the performance of the suboptimal distributed assignment is only slightly worse than the optimal performance, and, thus, outperforms existing distributed approaches by far.
\end{abstract}

\section{INTRODUCTION}

Cognitive Radio (CR) has become a popular and promising approach to overcome the artificial spectrum scarcity. The key idea of CR technologies is to allow the usage of temporarily unused licensed spectrum by so called Secondary Users (SUs) under the constraint that the spectrum has to be vacated, as soon as the owner of the band - referred to as Primary User (PU) - returns. To meet this constraint, the spectrum has to be sensed periodically - at least every $t_{\max }$ - to detect potentially appearing PUs. In order to perform reliable sensing on a frequency, data transmission has to be interrupted. Depending on the PU detection requirements and the sensitivity of the sensing antenna, the sensing process can require up to hundreds of milliseconds. Obviously, such interruptions in data transmission severely degrade the Quality of Service (QoS) especially for real-time or streaming applications.

To avoid periodic interruptions of the payload communication, Dynamic Frequency Hopping (DFH) has been proposed for cellular CR networks [1], [2]. The basic idea of DFH is the following: A cell performs sensing on frequency $Y$ in parallel to data transmission on frequency $X$. After $t_{\max }$, the cell hops to frequency $Y$ and performs sensing on frequency $X$ and so on. Obviously, this implies that sending and sensing in parallel is possible, e.g. by using two antennas. Having a whole network of mutually interfering cells, mitigating Co-Channel Interference (CCI) becomes crucial. The question is, how to

\footnotetext{
*Since January 2008, James Gross is with the Mobile Network Performance Group, UMIC Research Centre, RWTH Aachen University, gross@umic.rwth-aachen.de.
}

find a frequency assignment such that, (a) two interfering cells never use the same frequency at the same time, and (b) the total number of frequencies used in the network is minimized. Reducing the number of frequencies increases the number of supportable cells and additionally reduces the probability of interference with PUs.

The so called Frequency Assignment Problem (FAP) [3] is a well investigated topic for frequency-static (i.e. non-hopping) networks. Mathematically, the FAP can be expressed as a graph coloring problem with nodes (representing the cells) and edges between the nodes (representing their interference relationships). Each node is assigned one (or multiple) color(s) such that two connected nodes never own the same colors. Minimizing the total number of colors is - mathematically similar to the list coloring problem, which is known to be $n p$ complete [4]. However, in non-hopping networks, distributed heuristics - such as the Distributed Largest First (DLF) algorithm [5] - have been shown to achieve remarkably good results compared to the optimum for a wide set of graphs [6], while greatly reducing the computational complexity.

In this paper, we present a new concept for DFH called Double Hopping (DH) and compare it to previously suggested DFH approaches. We introduce a distributed heuristic called Distributed Frequency Assignment (DFA) and compare it to the Distributed Hopping Approach (DHA), introduced in [7]. Additionally, an optimal algorithm based on solving a Linear Integer Program (LIP) called Optimal Frequency Assignment (OFA) is developed to serve as a lower bound for frequency usage. The remainder of this paper is structured as follows: Section II presents related work on frequency hopping. The FAP and the system model is presented in Section III. The general DH approach is introduced in Section IV and the centralized and distributed algorithms in Section V. In Section VI, we present the performance evaluation results. Finally, in Section VII, we conclude the presented work.

\section{RELATED WORK}

\section{A. Frequency hopping}

The idea of frequency hopping has gained lots of attention in the context of Global System for Mobile Communications (GSM), Bluetooth ${ }^{\circledR}$, and Wireless Local Area Networks (WLANs). The main objective to apply frequency hopping in these systems is to mitigate fast fading and CCI. Hopping sequence design for GSM is studied in [8], [9]. Dynamic frequency hopping in GSM is studied and compared to random 
hopping in [10]. Here, the frequency hopping pattern of a mobile is adapted based on measurements made at the base station and the mobile. In Bluetooth ${ }^{\circledR}$, each cell chooses one out of several pre-specified pseudo-random hopping sequences. Recently, a non-cooperative Adaptive Frequency Hopping (AFH) method has been proposed to combat the so called frequencystatic interference that originates e.g. from WLAN systems or microwave ovens [11], [12]. In [13], Mishra et al. propose to assign each WLAN of a large, uncoordinated network a hopping pattern, such that the performance degradation of interference is somewhat spread over all cells in the network over a longer time span, leading to an increased system-wide cell-level fairness.

In contrast to the application of frequency hopping in nonCR related networks as described above, the scope of applying frequency hopping in CR networks is to allow continuous data transmission and at the same time to assure the unimpaired operation of the PU. To the best of our knowledge, so far, among existing frequency hopping applications solely Bluetooth ${ }^{\circledR}$ 's Adaptive Frequency Rolling (AFR) approach [14] is somewhat related and could be modified for CR operation. AFR tries to avoid frequencies occupied by WLAN systems (which could be seen as PUs) and at the same time also avoids CCI between different Bluetooth systems. However, since AFR has been developed under non-CR related assumptions, a high number of modifications would be necessary to support CR operation.

Frequency hopping in $\mathrm{CR}$ networks has been first considered within the IEEE 802.22 standardization process [15]. Based on that standard, we have been the first ones to introduce the general concept of Dynamic Frequency Hopping (DFH) in 802.22 [1], [2]. In these papers, we present phase-shifted operation for interference-free sensing and collision-free hopping in combination with a cooperative hopping approach for neighboring cells referred to as Revolver Hopping (RH). Additionally, the concept of Dynamic Frequency Hopping Community (DFHC) is proposed as an effective way to organize the hopping of neighboring cells. Based on the $\mathrm{RH}$ approach presented in [2], the Distributed Hopping Approach (DHA) is proposed in [7]. DHA is a distributed algorithm realizing RH in a cluster of IEEE 802.22 cells without the need of a central controller managing the hopping patterns of the individual cells. However, it is also shown in [7] that there is a large difference in the performance of DHA and the theoretical optimal application of RH. This circumstance was one of the main motivations for us to search for a better performing distributed solution for solving the frequency assignment problem, which is done in this paper.

\section{B. Graph coloring}

The utilization of graph coloring in order to find optimal frequency assignments in "frequency-static" networks is well documented in literature. Basic studies on its use in connection with cellular networks are reported in [16], [17]. For more indepth studies, there is a web page maintained by Eisenblätter and Koster [3].
In [18] and [19] two different approaches are made to use graph coloring in CR networks. However, note that in both papers, "frequency-static" networks are considered. In [18], in contrast to our approach, each node in the graph represents one CR terminal, which is subject to an individual PU interference. According to the experienced interference level, the frequencies differ in the reward accredited to the terminal in case of an assignment. In addition, power control is used to control interference. The objective in [18] is the maximization of the network utility subject to reward and fairness constraints for a given number of terminals and available frequencies. Recall that, in contrast to that, our goal is to minimize the number of necessary frequencies. In [19], the maximization of the network utility is considered as well, but, in contrast, all terminals experience the same interference from PUs. In conformity with our approach, the total number of frequencies used in the network is minimized. However, since there is a different graph coloring / network relation, and since the channels might differ in bandwidth, a different optimization problem is formulated.

\section{SYSTEM MODEL}

We consider a spectrum range of $B \mathrm{MHz}$, divided into $F_{\text {tot }}$ frequency bands of equal size, indexed from 1 to $F_{\text {tot }}$. Each of the frequency bands is owned by a PU. The total number of CR cells in the investigated network is denoted by $|V|$. Each cell consists of one Base Station (BS) and a number of associated terminals. We assume that user data sent under the impact of interference is lost. Two CR cells are interfering if both operate in the same frequency band at the same time (referred to as working frequency) and are within each others interference range. In the following, we refer to these cells as neighboring cells. Neighboring cells have means to exchange control information using a control channel. The control channel can be physically separated from the data transmission channel (e.g. using Ultra-Wideband (UWB) as proposed in [20], [21]) or can be a logical in-band control channel (e.g. as envisioned by the 802.22 working group [15]). In order to focus on the performance of the frequency assignment algorithms (and not the reliability of the control channel), we do not consider the specifics of the implementation of the control channel in this paper. We assume the lower layers of the control channel to provide a reliable message delivery service to our control communication protocols. A CR cell can, thus, learn the interference relationships within its neighborhood by receiving control messages from its neighbors. Interference topology graphs are used to model the interference relationships among cells. We define an interference topology graph $G=(V, E)$, where $V=\left\{v_{1}, \ldots, v_{n}\right\}$ denotes the CR cells and $E$ is the set of interference relationships with $(i, j) \in E$ if $v_{i}$ and $v_{j}$ are in each others' interference range. Additionally, we define $N_{i}$ to be the set of neighboring cells of $i$, i.e. $j \in N_{i}$ if $(i, j) \in E$.

The maximum time span an SU is allowed to interfere with a PU is referred to as $t_{\max }$. The data transmission time $\left(t_{\text {data }}\right)$ is the maximum period of time a CR cell consecutively uses a working frequency for data transmission, with $t_{\text {data }} \leq t_{\max }$. The 


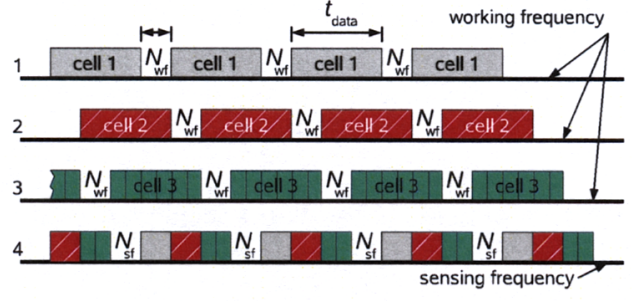

Fig. 1. Double Hopping operation

sensing time $\left(t_{\text {sens }}\right)$ is the minimum amount of time required to (1) perform sensing ${ }^{1}$ (by the BS and the terminals), (2) gather the sensing information at the BS, and (3) inform all terminals about the working frequency to be used for the next data transmission period. The quiet time $\left(t_{\text {quiet }}\right)$ is the amount of time a frequency is not used for data communication in order to perform sensing, which obviously implies $t_{\text {quiet }} \geq$ $t_{\text {sens. }}$. Note that we consider time to be discrete and quantized into units of $t_{\text {quiet }} . N_{q}$ is defined as the number of quiet times per data transmission time $\left(N_{q}=t_{\text {data }} / t_{\text {quiet }}\right)$.

Each CR entity is equipped with two antennas such that spectrum sensing and data transmission in parallel is possible. However, in order to perform reliable sensing, the frequency being sensed must not be used for data transmission. We do not investigate specific sensing mechanisms. Consequently, the reliability of sensing is not taken into account. Primary User (PU) interference is global, i.e. an appearing PU is likewise present in all cells of the network. Furthermore, we assume that PUs do not change their frequency usage frequently over time. While this static PU model is valid for certain scenarios (e.g. 802.22 in the TV bands), it mainly serves the goal of analyzing the theoretical potential of the presented algorithms. Dynamic and locally visible PUs are subject to future work.

\section{Double Hopping}

Double Hopping (DH) relies on the phase shifted operation of neighboring cells as introduced in [2]. Each cell exclusively uses a dedicated working frequency. Additionally, there is one sensing frequency used by all cells. Once the data transmission time $\left(t_{\text {data }}\right)$ of a cell expires, the cell hops to the sensing frequency, in order to perform sensing on its working frequency. It continues its communication on the sensing frequency for $t_{\text {quiet }}$, before hopping back to its working frequency. Due to the time shifted operation, all cells can consecutively switch to the sensing frequency to perform sensing on their working frequency. After one cycle, the sensing frequency has to be sensed simultaneously by all cells. Consequently, each cell needs two sensing slots, one for the working frequency $\left(N_{\mathrm{wf}}\right)$ and one (shared by all cells) for the sensing frequency $\left(N_{\mathrm{sf}}\right)$. Figure 1 shows three neighboring cells performing $\mathrm{DH}$.

As a consequence of the phase-shifted operation, the maximum number of neighboring cells that can be supported in hopping mode is the number of sensing slots per data

\footnotetext{
${ }^{1}$ The time needed for sensing depends on the chosen sensing technique (which is beyond the scope of this paper) and on the number of frequencies to sense. Note that we assume that all frequencies can be sensed in parallel.
}

transmission time $\left(N_{q}\right)$. Thus, if $M$ neighboring cells with $M>N_{q}$ have to be coordinated at once, $M-N q$ cells have to operate in a different frequency range. We assume those cells to operate in non-hopping mode, i.e. they periodically interrupt data transmission to perform sensing.

The most significant difference between DH and Revolver Hopping (RH) (as described in [2], [7]) is the frequency requirement per cell: while the overall minimum frequency requirement is the same in both approaches, the frequency requirement per cell is much larger in the RH approach: each cell hops through the whole set of used frequencies. In the DH approach, in contrast, a CR cell hops between two frequencies only. This has several advantages: (1) The sensitivity to PU interference is smaller: since each working frequency is exclusively used by one cell, solely this cell has to be shifted to another frequency in case a PU appears. (2) Managing the coordination between different cells is easier: Cells of one hopping community only share the sensing frequency (compared to the whole set of used frequencies in the RH approach). (3) The number of blocked frequencies per cell is smaller in the DH approach, i.e. the chance for spatial frequency reuse in adjacent hopping communities is bigger.

\section{HOPPING PATTERN GENERATION}

In this section, we formulate two graph coloring based alternatives to generate hopping patterns that minimize the number of frequencies (colors) in use and ensure a collision free assignment of frequencies among neighboring cells (nodes): an optimization based, centralized approach and a distributed heuristic. Note that we use the optimal approach as a performance comparison reference rather than proposing it for use in practice.

\section{A. Optimal Frequency Assignment}

We assume one central entity to be responsible for the DH pattern generation of all cells in the network. The central entity has global knowledge about the interference graph. Optimization tools are used to assign optimal hopping patterns by solving LIP (1). We refer to this approach as Optimal Frequency Assignment (OFA).

$$
\begin{array}{lll}
\min & \sum_{\forall c \in C} b_{c}+\frac{1}{|C|} \sum_{\forall c \in C} a_{c} & \\
\text { s.t. } & \sum_{\forall c \in C} x_{c, v}=1 & \forall v \in V \\
& x_{c, v}+x_{c, w} \leq 1 & \forall c \in C \wedge \forall(v, w) \in E \\
\sum_{\forall c \in C} y_{c, v}=1 & \forall v \in V \\
x_{c, v}+y_{c, v} \leq 1 & \forall(c, v) \in C \times V \\
x_{c, v}+y_{c, w} \leq 1 & \forall c \in C \wedge \forall(v, w) \in E \\
y_{c, v}+\sum_{\forall w \in N_{v}} y_{c, w} \leq N_{q} & \forall(c, v) \in C \times V \\
a_{c} \geq x_{c, v} & \forall(c, v) \in C \times V \\
b_{c} \geq y_{c, v} & \forall(c, v) \in C \times V
\end{array}
$$




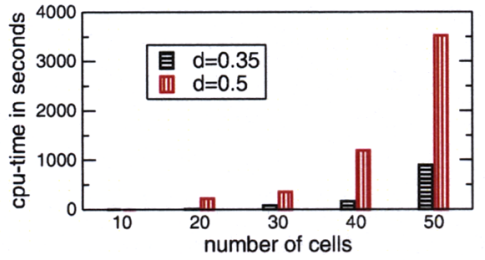

Fig. 2. CPU times for LIP solving using CPLEX on a Pentium $43.20 \mathrm{GHz}$

Here $x_{c, v}$ and $y_{c, v}$ are the binary assignment variables with

$x_{c, v} \begin{cases}=1 & \text { if node } v \text { uses color } c \text { as working color, } \\ =0 & \text { if node } v \text { does not use color } c \text { as working color, }\end{cases}$

and

$y_{c, v} \begin{cases}=1 & \text { if node } v \text { uses color } c \text { as sensing color, } \\ =0 & \text { if node } v \text { does not use color } c \text { as sensing color. }\end{cases}$

Assuming the interference graph $G$, solving the LIP assigns each node $v \in V$ a working and a sensing color $c_{w}, c_{s} \in C$. Constraint (1b) assures that exactly one working color is assigned to each node, and constraint (1c) assures that neighboring nodes do not get the same working color. Constraint (1d) assures that exactly one sensing color is assigned to each node, constraint (1e) assures that the sensing color of a node differs from its working color, and constraint (1f) assures that the sensing color differs from the working colors of neighboring nodes. Constraint (1g) ensures that no more than $N_{q}$ neighboring nodes share the same sensing color. To indicate whether color $c$ is used by any node in the graph, the auxiliary variables $a_{c}$ and $b_{c}$ are defined by constraints (1h) and (1i). Consequently, they are the variables to be minimized in the optimization goal (1a), where minimization of the sensing colors $\left(\sum b_{c}\right)$ is the primary objective, and minimization of the working colors $\left(\sum a_{c}\right)$ is the secondary objective.

After solving the LIP, the central entity derives the working and sensing frequency, as well as the sensing slots for each cell from the assignment variables $x$ and $y$ : Each cell $v$ with $x_{c, v}=1$ gets assigned the frequency with index $c$ as working frequency and $N_{\mathrm{wf}}=c$ as sensing slot for the working frequency. Additionally, each cell $v$ with $y_{c, v}=1$ gets assigned the frequency with index $c$ as sensing frequency, and the $N_{\text {sf }}=c$ as sensing slot for the sensing frequency. The frequency assignment information has to be distributed among all cells in the network. Additionally, the central entity has to recompute and redistribute the assignment as soon as there is any change in the interference relationship between the cells in the graph or as soon as a used frequency cannot be used anymore due to the appearance of a PU. To keep the view on the interference graph up to date at the central entity, all cells in the network have to periodically report their local view on the interference graph to the central entity.

The message overhead required per cell to collect and periodically update the information about the whole interference graph at the central entity and to distribute the frequency assignments to the individual cells increases linearly with the average number of hops to reach the central entity. Addition- ally, solving the above LIP and, thus, the computation of the optimal frequency assignment, might require extremely long computation times. Figure 2 shows the average run-times of an Intel Pentium $43.20 \mathrm{GHz}$ solving the above LIP as part of our simulations using the CPLEX [22]. The expected computation time increases exponentially with the number of nodes in the graph, which poses some scalability concerns.

\section{B. Distributed Frequency Assignment}

Distributed Frequency Assignment (DFA) is an efficient heuristic for DH pattern generation. In contrast to the optimal approach above, its computational complexity is low and it requires a constant message overhead, which is independent of the network size. Each cell holds information about its neighborhood only: It learns the frequency usage of its neighbors by exchanging control messages on the control channel.

DFA is based on the Distributed Largest First (DLF) graph coloring algorithm [5] originally designed to solve static FAPs, which performs near to optimal in practical problem instances [6]. The basic idea of DLF is the following: Each node of the graph determines and broadcasts its node degree (number of neighboring nodes) and collects information about its neighbors' node degrees. A node does not choose its working color until having received the decision of all neighboring nodes with a higher node degree. It then chooses the color with the lowest index available and broadcasts its decision. In the case of equal node degrees, random numbers are used for tie breaking. This ensures that two neighboring nodes cannot select the same color (as only one color is chosen at a time).

Since DH requires a sensing frequency and sensing slots in addition to the working frequency for each cell, the DLF has to be modified accordingly: When applying DFA, the number of the sensing slot for the working frequency $\left(N_{\mathrm{wf}}\right)$ is equal to the working frequency index. Additionally, each cell selects a sensing frequency and sensing slot for the sensing frequency $\left(N_{\mathrm{sf}}\right)$ before broadcasting its frequency usage. If possible, the cell reuses a sensing frequency and $N_{\text {sf }}$, which is used and has been announced by a neighboring cell. If no sensing frequency has been announced or the announced sensing frequencies or slot cannot be used, it "creates" a new one. Once all frequency and slot decisions have been made, they are broadcasted. Whenever a new cell pops up, it has to learn the frequency usage of its neighbors. It listens to control messages broadcasted by its neighbors for $t_{\text {data }}+t_{\text {quiet }}$ before choosing its frequencies and sensing slots according to the rules described above.

After sensing its working frequency (every $N_{\text {wf }}$ ), each cell broadcasts a message containing the updated frequency usage information. In case a PU is detected on the working frequency, a cell chooses a new working frequency (with the lowest frequency index available). If no new working frequency is available it switches to the non-hopping mode as previously described. Accordingly, a new sensing frequency needs to be selected and announced, if a PU is detected on the sensing frequency. A detailed description of the protocol realization and implementation can be found in [23]. 


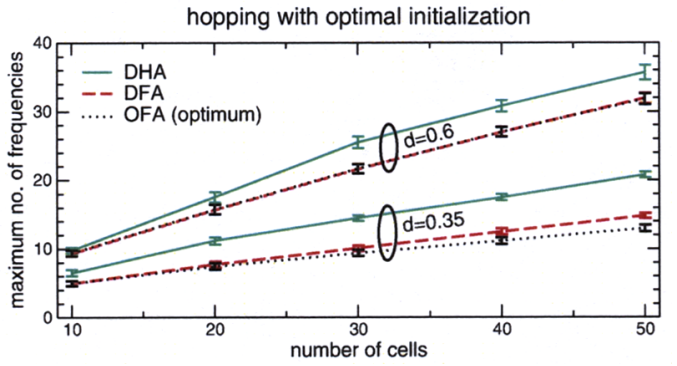

(a) The maximum number of frequencies required for $d=0.35$ and $d=0.6$ in the optimally initialized hopping case

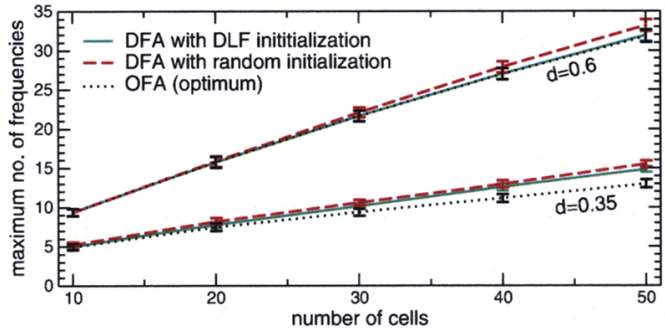

(c) The comparison of the maximum frequency requirement in case of DLF and sequential initialization for the DFA

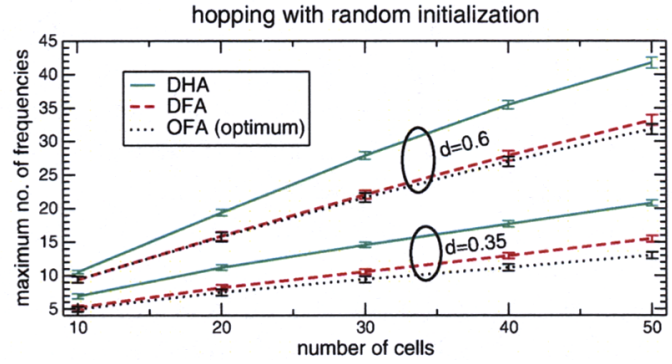

(b) The maximum number of frequencies required for $d=0.35$ and $d=0.6$ in the randomly initialized hopping case

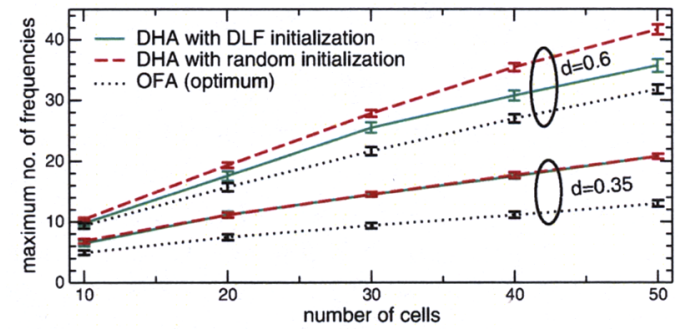

(d) The comparison of the maximum frequency requirement in case of DLF and sequential initialization for the DHA

Fig. 3. Performance evaluation results (averaged over all instances)

\section{PERFormance ANALYSiS}

\section{A. Methodology}

We have randomly generated interference topology graph instances using Culbersohn's graph generator [24] on a 1 by 1 unit plane, with the number of nodes varying between $|V|=10$ and $|V|=50$. Two nodes are within each others' interference range if their euclidian distance is smaller than or equal to $d$, where we vary this distance between $d=0.35$ and $d=0.6$. We have generated 80 random graph topologies for each of the $(|V|, d)$ pairs. The simulated system comprises a total number of $F_{\text {tot }}=50$ frequencies, where frequencies 1 to 30 are used for the hopping mode and frequencies 31 to 50 are used for the non-hopping mode. This concept enables us to support up to $2 N_{q}$ neighboring CR cells in time shifted operation, i.e. in a collision-free operation (assuming that the exchange of control messages is reliable). The simulation time for each simulation is set to $200 \mathrm{~s}$. The quiet time is set to $t_{\text {quiet }}=0.1 \mathrm{~s}$, the data transmission time is set to $t_{\text {data }}=1.9 \mathrm{~s}$. Based on the generated graph topologies, the dynamic frequency selection and communication between the cells is simulated using the timed discrete event system simulator OMNeT++ [25] including the Mobility Framework [26].

We investigate two initialization methods. In the optimal initialization, all cells start operation synchronously using an initial hopping pattern determined using the DLF. In the random initialization, a cell randomly starts operation within the first 10 seconds of the simulation. We compare the performance of the Distributed Frequency Assignment (DFA) introduced in this paper with the Distributed Hopping Approach (DHA) from [7]. The Optimal Frequency Assignment (OFA) is used as a lower bound to benchmark the performance.

\section{B. Optimal initialization results}

In this section we compare the number of frequencies used assuming a synchronous start of the cells and using a pre-calculated initial coloring. In Figure 3(a) we show the maximum number of frequencies required per run, averaged over all runs. The figure shows that the DFA approach achieves remarkably good results. For $d=0.6$ there is almost no difference between the DFA and the optimal OFA. This is mainly due to the fact that for the DFA approach the initial coloring computed by the DLF is kept as long as there are no changes in the interference environment (i.e. no PU or no additional CR shows up). As a consequence, the performance only depends on the initial coloring, which is nearly optimal for the investigated graph instances using the DLF algorithm. In the DHA case however, a lot more frequencies are required compared to the OFA results. Each cell individually chooses a new frequency (with the lowest index available) before it has to perform a hop, which does not always result in periodic hopping pattern and, thus, might lead to a sub-optimal frequency usage.

\section{Random initialization results}

In this simulation scenario we investigate the number of frequencies used by DFA and DHA assuming a sequential random initialization of cells. Figure 3(b) shows the average of the maximum number of frequencies required over the number of cells in the network. The bottom three graphs show the results for $d=0.35$, the top three graphs for $d=0.6$. The figure shows that the DFA clearly outperforms the DHA also in the case where no initial coloring is used. Moreover, the results achieved by the DFA approach can compete with the optimal solution, especially for small $|V|$. The difference between 
the optimal solution and the DFA approach is smaller for $d=0.6$ (in accordance with the optimal initialization results). In Figure 3(c) and 3(d) we compare the results achieved in case of DLF initialization and random initialization for the DFA and the DHA respectively. For the DFA we can see that the difference is marginal, i.e. even using a non-optimal initialization solely based on the (random) appearance of the cells achieves good results. For the DHA it is interesting to observe, that for $d=0.35$ there is no difference between the DLF and the optimal initialization. For $d=0.6$, however, the figure shows that the performance difference increases as the number of cells in the network increases.

\section{CONCLUSIONS}

In this paper we have presented Double Hopping (DH), a new approach to apply Dynamic Frequency Hopping (DFH) in CR cellular networks. The presented approach allows for continuous data transmission in CR networks while enabling reliable detection of PUs. We have presented two frequency assignment alternatives for $\mathrm{DH}$, which minimize the total number of frequencies used: centralized Optimal Frequency Assignment (OFA) and Distributed Frequency Assignment (DFA). Minimizing the number of required frequencies in DFH cellular CR networks has the goal of (a) minimizing potential SU generated interference on PUs, and (b) maximizing the number of supportable CR cells. Our performance evaluation results show that the proposed DFA heuristic can compete with the optimal results of the OFA while significantly reducing the computational complexity and control message overhead. We, thus, have shown that - as in the frequency-static case - distributed approaches for solving the FAP exist, which achieve results comparable to the optimum.

Investigating the impact of PU dynamics is left as a future work issue. Another issue is to explore the impact of the amount of neighborhood information (i.e. the frequency usage of neighboring cells) on the two approaches. Currently, each cell has knowledge about its one-hop neighborhood only. The related research question to answer is if the approaches significantly gain from e.g. having two-hop neighborhood knowledge. Additionally, our preliminary results motivate the introduction of cooperation between hopping cells by grouping cells into communities. Each community can be assumed to have regional information about its vicinity. The trade-off between the overhead to keep these informations up to date and the gain in performance is another interesting optimization problem.

\section{REFERENCES}

[1] L. Chu, W. Hu, G. Vlantis, J. Gross, M. Abusubaih, D. Willkomm, and A. Wolisz, "Dynamic frequency hopping community," online, IEEE 802.22 Working Group, Technical proposal submitted to IEEE 802.22 WG 22-06-0113, Jun. 2006.

[2] W. Hu, D. Willkomm, L. Chu, M. Abusubaih, J. Gross, G. Vlantis, M. Gerla, and A. Wolisz, "Dynamic frequency hopping communities for efficient IEEE 802.22 operation," IEEE Commun. Mag., Special Issue: "Cognitive Radios for Dynamic Spectrum Access", vol. 45, no. 5, pp. 80-87, May 2007.

[3] A. Eisenblaetter and A. Koster, "FAP web page," 2000. [Online]. Available: http://fap.zib.de
[4] K. H. Rosen, Discrete Mathematics and Its Applications. McGraw-Hill Higher Education, 1998.

[5] M. Kubale and L. Kuszner, "A better practical algorithm for distributed graph coloring," in Proceedings of the IEEE International Conference on Parallel Computing in Electrical Engineering 2002 (PARELEC 02). IEEE, 2002, pp. $72-76$.

[6] J. Hansen, M. Kubale, L. Kuszner, and A. Nadolski, "Distributed largestfirst algorithm for graph coloring," in Proceedings of the 10th International Euro-Par Conference, vol. 3149/2004. Pisa, Italy: Springer Berlin / Heidelberg, Aug. 2004.

[7] D. Hollos, D. Willkomm, J. Gross, and W. Hu, "Centralized vs. distributed frequency assignment in frequency hopping (cognitive radio) cellular networks," Telecommunication Networks Group, Technische Universität Berlin, Tech. Rep. TKN-07-007, Dec. 2007.

[8] P. Björklund, P. Värbrand, and D. Yuan, "Optimal frequency planning in mobile networks with frequency hopping," Computers and Operations Research, vol. 32, pp. 169-186, 2005.

[9] J. Moon, L. Hughes, and D. Smith, "Assignment of frequency lists in frequency hopping networks," IEEE Trans. Veh. Technol., vol. 54, no. 3, pp. 1147-1159, 2005.

[10] Z. Kostic, I. Maric, and X. Wang, "Fundamentals of dynamic frequency hopping in cellular systems," IEEE J. Sel. Areas Commun., vol. 19, no. 11, pp. 2254-2266, Nov. 2001.

[11] M. Cho-Hoi Chek and Y.-K. Kwok, "On adaptive frequency hopping to combat coexistence interference between bluetooth and IEEE 802.11b with practical resource constraints," in Proceedings of the 7th International Symposium on Parallel Architectures, Algorithms and Networks, 2004, pp. 391-396.

[12] B. Zhen, Y. Kim, and K. Jang, "The analysis of coexistence mechanisms of bluetooth," in Preceedings of the 55th IEEE Vehicular Technology Conference, 2002. (VTC Spring 2002), vol. 1, May 2002, pp. 419-423.

[13] A. Mishra, V. Shrivastava, D. Agrawal, S. Banerjee, and S. Ganguly, "Distributed channel management in uncoordinated wireless environments," in Proceedings of the 12th annual international conference on Mobile computing and networking (MobiCom 2006). New York, NY, USA: ACM, 2006, pp. 170-181.

[14] H. Yomo, P. Popovski, H. Nguyen, and R. Prasad, "Adaptive frequency rolling for coexistence in the unlicensed band," IEEE Trans. Wireless Commun., vol. 6, no. 2, pp. 598-608, 2007.

[15] “IEEE 802.22 working group." [Online]. Available: http://www.ieee802. org $/ 22$

[16] B. Sanso and P. Soriano, Eds., Telecommunications Network Planning. Norwell, MA, USA: Kluwer Academic Publishers, 1998.

[17] A. Koster, "Frequency assignment: Models and algorithms," Ph.D. dissertation, Universiteit Maastricht, 1999.

[18] C. Peng, H. Zheng, and B. Y. Zhao, "Utilization and fairness in spectrum assignment for opportunistic spectrum access," Mobile Networks and Applications, vol. 11, no. 4, pp. 555-576, 2006.

[19] S. Sengupta, S. Brahma, M. Chatterjee, and S. Shankar N, "Enhancements to cognitive radio based IEEE 802.22 air-interface," in Proceedings of the IEEE International Conference on Communications, 2007 (ICC '07), 24-28 June 2007, pp. 5155-5160.

[20] R. W. Broderson, A. Wolisz, D. Cabric, S. M. Mishra, and D. Willkomm, "CORVUS: A cognitive radio approach for usage of virtual unlicensed spectrum," White Paper, 2004.

[21] D. Cabric, S. M. Mishra, D. Willkomm, R. W. Broderson, and A. Wolisz, "A cognitive radio approach for usage of virtual unlicensed spectrum," in 14th IST Mobile Wireless Communications Summit 2005, Dresden, Germany, June 2005.

[22] S. ILOG, “CPLEX solver," 2007, (accessed 04. Mai 2007). [Online]. Available: http://www.ilog.fr/products/cplex/

[23] D. Willkomm, M. Bohge, D. Hollos, and J. Gross, "Double hopping: A new approach for dynamic frequency hopping in cognitive radio networks," Telecommunication Networks Group, Technische Universität Berlin, Tech. Rep. TKN-08-001, Jan. 2008.

[24] J. Culberson, A. Beacham, and D. Papp, "Hiding our colors," in CP'95 Workshop on Studying and Solving Really Hard Problems, Cassis, France, 1995, pp. 31-42.

[25] A. Varga, OMNeT++ Discrete Event Simulation System. [Online]. Available: http://www.omnetpp.org/doc/manual/usman.html

[26] "Mobility framework (MF) for simulating wireless and mobile networks using OMNeT++." [Online]. Available: http://mobility-fw.sourceforge. net/ 